\title{
Radiationless transitions and photochemical reactivity
}

\author{
Sebastiăo J. Formosinho \\ Departamento de Química, Universidade de Coimbra \\ 3049 COIMBRA Codex, Portugal
}

\begin{abstract}
The potential energy along a reaction coordinate can be calculated in terms of the reaction energy, the force constants and the equilibrium bond lengths of the reactive bonds, the bond order at the transition state and, for very exothermic or endothermic reactions, the so called "configuration entropy". Such potential energy curves allows one to assess the importance of quantum mechanical tunnelling in $\mathrm{H}-$ abstraction, $\alpha$-cleavage and cycloaddition photochemical reactions of excited ketones. Experimental recognition of tunnelling can be made by the study of the efficiency of the photoreactions as a function of molecular parameters and medium properties. The theory allows the establishement of structure-efficiency relationships and criteria to distinguish between the mechanisms of nuclear tunnelling and thermal activation.
\end{abstract}

\section{INTRODUCTION}

Despite the high energy of excited states, these molecular species do not react indiscriminately but are controlled by the same forces as ground state species and possibly by the same kind of potential energy barriers. Although the best procedure to seek for relationships between molecular structure and chemical reactivity of ground and excited states is the construction of potential energy surfaces, at present this is an impossible task in organic photochemistry. Neverthless a considerable insight on chemical reactivity can be obtained from the knowledge of the energy change along reaction coordinates. In the past years there has been a renewed interest in understanding how the harrier of a chemical reaction depends on the reaction energy, $\Delta E$, and in elucidating the position of the energy barrier (1-3). The current approaches, which have an empirical character, are generalizations of the well known Marcus - equations (4). These approaches (2) measure the extent to which $\Delta E$ propagates across the reaction coordinate and the height and shape of the barrier for the hypothetical thermoneutral reaction.

\section{A GENERAL INTERSECTING-STATE-MODEL FOR REACTION ENERGY BARRIERS}

In collaboration with Professor Varandas, we have recently developed a semi-empirical approach to estimate the energy barrier of chemical reactions in terms of parameters outside the fields of chemical kinetics (5). For the hypothetical reaction $A+B C \rightarrow A B+C$ the energy barrier is estimated by the intersection of the potential energy curves (e.g. harmonic or Morse oscillators) of $A B$ and $B C$. The height of the energy barrier, depends on the shape of the potential energy curves, on the reaction energy and on the distance, d, between the minima of the potential energy curves of reactant and product. This parameter corresponds also to the sum of the bond distensions to the transition state. The theory shows that

$$
d=n\left(I_{A B}{ }^{+I} B C\right)
$$

where $I_{A B}$ and $I_{B C}$ are the equilibrium bond lengths of $A B$ and $B C$ and $\eta$ is the reduced bond distension,

$$
\eta=\frac{a^{\prime} \ln 2}{n^{\ddagger}}+\frac{a^{\prime}}{2 \lambda^{2}}(\Delta E)^{2}
$$

$\mathrm{n}^{\ddagger}$ is the average bond order of the transition state, $\lambda$ is the "configuration entropy" parameter as defined by Agmon and Levine (6), and $a^{\prime}$ is a constant ( $\left.a^{\prime}=0.156\right)$.

The present model can assess independently the role of several thermodynamic, geometric and electronic factors on $\Delta E^{\ddagger}$, namely $\Delta E$, the force constants of the reactive bonds $(f)$, or other characteristics of the potential energy curves, the sum of lengths of the reactive bonds (I), the bond order of the transition state $\left(n^{\ddagger}\right)$ and the configuration entropy $\lambda . \Delta E, I$ and $f$ can be known from thermodynamic and spectroscopic data and for many reactions $n^{\ddagger}$ can be obtained from molecular orbital theory (7). The configuration entropy, however, has to be treated as an empirical parameter, which was found to be proportional to the activation entropy; $\lambda$ is high when $\Delta S^{\ddagger}$ is highly positive and $\lambda$ is low when $\Delta S^{\neq}<0$ (7).

The present model includes the Marcus (4) and the BEBO (8) models as particular cases. The Marcus model corresponds to the situation $\lambda \gg>\triangle E$ and the intrinsic energy barrier $\Delta E^{\neq}(0)=(1 / 8) f\left(a^{\prime} \ln 2\left(I_{A B} B_{B C}\right) / n^{\neq} \mid 2\right.$ The model introduces the concept of variable bond order along the reaction coordinate due to siphoning 
of electron density from nonbonding, antibonding electrons or from substituent groups into the transition state $(5,7)$; the BEBO model corresponds to the particular case where the total bond order is conserved. Furthermore the model also includes the Woodward-Hoffmann rules (9), because a symmetry forbidden reaction has $n^{\ddagger}=0$ and an energy barrier equal to the dissociation energy of the reactant $\mathrm{BC}$. The model incorporates also features of the variational versions of the Transition State Theory (10). In eq (2) the first term localizes the "saddle point" on the reaction coordinate; the second term takes into account the possibility that the "transition state" may not coincide with the "saddle point" due to entropic factors.

Modern semi-empirical models try to circumvent the stiffness of the Marcus-equations by assuming two or more independent variables to define the progress of reaction for a reaction series $(2,3,11)$, in spite of the fact that this situation bears no resemblanceto potential energy surfaces current in molecular dynamics (12). The present model avoids such difficulties because it has only one progress variable and is considerably more flexible than the model of Marcus. For example, the model is capable of interpreting substituent effects in non-linear free-energy relationships such as with sigmatropic shifts and cycloaddition reactions (13), without involving parallel or perpendicular effects along the reaction coordinate. The model was also applied with succss to the study of several reactions which are thermally activated, namely radical reactions (5), electron transfer and nucleophilic substitutions (7).

\section{RADIATIONLESS TRANSITIONS AS NUCLEAR TUNNELLING}

In addition to several radiative and nonradiative physical deactivation processes, an electronic excited state can sometimes undergo chemical reactions through large changes of nuclear configurations. The escape of the chemical species from their minima of potential energy can occur over the energy barrier via thermal activation, or through the barrier via a radiationless process. Therefore, theories of nonadiabatic multiphonon radiationless processes can provide an adequate interpretation of photochemical reactions (14). Neverthless the description of molecular systems in classical terms offers significant advantages over quantum mechanics in terms of computations and physical insight into the dynamics of molecules. Because molecules obey quantum mechanics, qualitative and quantitative differences between classical and quantum dynamics become important and are generally described as "quantum effects". One of those effects is tunnelling. Jortner and UIstrup (14) have shown that for exoenergetic processes the multiphonon theories of radiationless transitions can be reduced to tunnelling expressions for Franck-Condon factors. Here we will follow this simpler approach, which has already proved to be able to interpret hydrogen photoabstraction $(15,16)$ and photoketonization reactions $(17)$, because it may allow the use of the same potential energy barriers for thermal activation and nuclear tunnelling mechanisms.

The rate of an adiabatic chemical reaction over a potential energy barrier of height $\Delta E^{\ddagger}$ is

$$
k_{\text {thermal }}=A c_{0}^{1-m} \exp \left(-\Delta E^{\ddagger} / R T\right)
$$

where $A$ is the preexponential factor, $c_{0}=1 \mathrm{M}$ and $\mathrm{m}$ is the molecularity of the reaction. The rate for the changes in nuclear configuration through the same barrier of harmonic oscillators

$$
k_{\text {tunnelling }}=\nu \exp \left[-\frac{2 \pi}{h} \quad\left(2 \mu \Delta E^{\ddagger}\right)^{1 / 2} \Delta x\right]
$$

where $v$ is an average frequency of the modes involved in the transition, $\mu$ is the reduced mass of the reactive oscillators, $\Delta x$ is the width of the energy barrier and the other symbols have their usual meaning. Although the relative importance of tunnelling versus thermal activation can only be assessed in quantitative terms, qualitatively we can except that tunnelling will predominate with light oscillators, and when the energy barrier is narrow and high. For the potential energy barrier calculated through eq (1) we expect that the latter situation will be favoured with oscillators of small bond lengths, high force constants and high bond orders at the transition state.

\section{HYDROGEN PHOTOABSTRACTIONS BY KETONES}

Hydrogen-abstraction by excited carbonyl compounds is a kind of photochemical reaction which seems to meet the conditions previously stated for a dominant tunnel effect. The intermolecular reactions are bimolecular processes which can be discussed under the present theoretical formalism in terms of "supermolecules". One only needs to postulate that motion along some direction in the configuration space of the system is slow and under this assumption $v$ is taken typically as a collision frequency $10^{11} \mathrm{M}^{-1} \mathrm{~s}^{-1}$. The vibrational modes which suffer large bond length changes are the $C=0^{*}$ and $C-H$ bonds in the reactants and the $\mathrm{C}-\mathrm{O}$ and $\mathrm{O}-\mathrm{H}$ modes in products. Consequently these are the reactive modes which will be used to construct the potential energy barrier.

Because there are two modes in reactants and in products, an effective force constant has to be calculated to treat the reaction on an unidimensional basis. The effective force constant (15) for an equal contribution of the two modes can be calculated as

$$
f_{\text {eff }} \simeq\left(f^{2} i+f^{2}\right)^{1 / 2}
$$

For intermolecular processes the effective force constants are $f_{r}=46.9 \times 10^{4} \mathrm{~cm}^{-1} A^{0-2}$ and $f_{p}=43.2$ $\times 10^{4} \mathrm{~cm}^{-1} A^{-2}$. The reaction energy is $-\triangle E=E_{e}+D(O H)-D(C H)-E_{\rho}$ where $E_{e}$ is the energy of the excited state, $D(X H)$ a bond dissociation energy and $E_{\rho}$ a term which takes into consideration the $\pi$ energy of the carbonyl group in the ground state and the reorganizational energy of the radical 
intermediates. For example $E_{\rho}$ was estimated as $22000 \mathrm{~cm}^{-1}$ for the reaction between benzophenone $3\left(n, \pi^{*}\right)$ and isopropanol (18). The effective reduced mass is $\mu^{1 / 2}=\mu^{1 / 2}+\mu^{1 / 2}$. The reactions from $n, \pi^{*}$ triplet and singlet states which will be discussed here are adiabatic processes (15).

The separation $d$ between the minima of the potential energy curves of reactants and products can be calculated through eqs (1) and (2). For the $\mathrm{XH}$ modes $\mathrm{I}_{\mathrm{CH}}=0.97 \mathrm{~A}^{\circ}$ and $\mathrm{I}_{\mathrm{OH}}=1.096 \mathrm{~A}$; ; since the bond order of each bond is $n=1$, assuming conservation of the bond order along the reaction coordinate, in a bond breaking bond-forming process $n^{\neq}=1 / 2$. With such set of values $d_{X H}=0.446 A^{\circ}$. The reaction involves also the $\mathrm{CO}$ modes. If the excited carhonyl compound is viewed as an alkoxy radical, the $\mathrm{C}-\mathrm{O}$ bond has $n=1$ and $I=1.426 A^{\circ}$ (force constants and bond lengths data from A. J. Gordon and R. A. Ford, "The Chemist's Companion", Wiley, 1972, p. 107 and 114). The same parameters are also employed for the radical intermediate. However, now the $\mathrm{C}-\mathrm{O}$ bond suffers no bond-breaking bond-forming process and consequently $n^{\ddagger}=1$. Then $d_{C O}=0.308 A^{\circ}$ and the value used in the calculation is the average of the two values, $d=\left(d_{C}-Q+d X-H\right) / 2$, i. e., $d=0.377 A$. The calculated rate constant for tunnelling for the $H$-abstraction of $3\left(n, \pi^{*}\right)$ benzophenone from isopropanol is $5.0 \times 10^{5} M^{-1} s^{-1}$, in excellent agreement with the experimental value $4.5 \times 10^{5} \mathrm{M}^{-1} \mathrm{~s}^{-1}$; other relevant data $\mathrm{D}(\mathrm{CH})=367.8 \mathrm{~kJ} \mathrm{~mol}-1, D(O H)=435 \mathrm{~kJ}$ $\mathrm{mol}^{-1}, \mathrm{E}_{\mathrm{e}}=287.8 \mathrm{~kJ} \mathrm{~mol}^{-1}(18)$. At the transition state we have neglected any resonance interaction which effectively decreases slightly the harrier height and slightly thickens the barrier at the top. For tunnelling this leads to a cancellation of two small errors.

The calculated barrier height is $54 \mathrm{~kJ} \mathrm{~mol}^{-1}$ which leads to a rate for thermal activation of $30 \mathrm{M}^{-1} \mathrm{~s}^{-1}$ with $A=10^{11} \mathrm{~s}^{-1}$, typical value for a bimolecular process. However for thermal reactions of ground state molecules in solution, the intersecting-state-model was found to reproduce the free energy barrier and not the activation energy. If for the thermal activated process the calculated energy barrier is a free energy curve, than the maximum preexponential factor is $k_{B} T / h=6 \times 10^{12} \mathrm{~s}^{-1}$. Under this assumption the thermal rate is $2 \times 10^{3} \mathrm{M}^{-1} \mathrm{~s}^{-1}$ still much smaller than the rate by tunnelling.

Many other alkoxy-type intermolecular and intramolecular $\mathrm{H}$-photoabstraction reactions by $1,3\left(n, \pi^{*}\right)$ and $3\left(\pi, \pi^{*}\right)$ carbonyl compounds with several substrates have been studied, and calculations support the conclusion that at room temperature tunnelling is always a few orders of magniture higher than thermal activation $(15,18)$. Reactions which have a strong charge-transfer character have $\mathrm{n}^{\neq}$values higher than the radical-type reactions and consequently have smaller $d$ values. For the fastest processes tunnelling and thermal activation have comparable magnitudes. Linear dependence of $d$ with the affinity of the ketones and the ionization energy of the substrates have been found (15). This is in accordance with the Schomaker and Stevenson relationship (19) which suggests that bond lengths are a funtion of the electronegativity difference between the bonded atoms.

Although calculations indicate that hydrogen photoabstractions can be viewed as radiationless transitions, it is important to confirm such a view experimentally. Recognition of tunnelling in chemical reactions is normally undertaken at very low temperatures, where tunnelling is revealed by rates which are virtually temperature independent. However large temperature changes affect the physical properties of the solvent medium and can also affect the kinetics of chemical reactions (20). Arnaut et al. (18) have shown that the study of quantum efficiencies under current experimental conditions provides a criterium to distinguish between the mechanisms of tunnelling and thermal activation.

Salem diagrams (21) for $\mathrm{H}$-abstractions reveal the existence of a crossing point $(\omega)$ between the ground state and the radical intermediates, I, potential energy curves. The energy content of the intermediates is different for tunnelling or thermal activation mechanisms. For the latter mechanisms, the point $\omega$ will always be found along the reaction coordinate, and a crossing $I m \rightarrow S_{0}$ will decrease the efficiency of the radical formation, $\phi_{\mathbf{r}}<1$ (Figure 1). However for a tunnelling mechanism the same situation only arises when $\omega$ is below the reactive excited state, $i$. e., $E_{\omega}<E\left(T_{1}\right.$ or $\left.S_{1}\right)$; when $E_{\omega}>E\left(T_{1}\right.$ or $\left.S_{1}\right)$ the crossing point will not be found along the reaction coordinate and $\phi_{r}=1.0$. Table 1 confirms such prediction for several intermolecular reactions of $3\left(n, \pi^{*}\right)$ states. The energy of the crossing point was

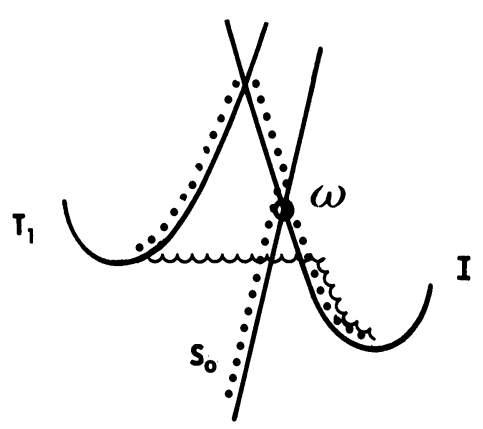

Fig. 1. Nuclear configuration diagrams for $\mathrm{H}$-photoabstraction reactions

-...thermal activation;

nuclear tunnelling.
TABLE 1. Efficiencies of intermolecular hydrogen abstractions by ${ }^{3}\left(n, \pi^{*}\right)$ ketones and phenones 호.

\begin{tabular}{|c|c|c|c|}
\hline Ketone & Substrate & $\mathrm{E}_{\omega}-\mathrm{E}_{\mathrm{T}_{1}} / \mathrm{cm}^{-1}$ & $\phi$ \\
\hline Benzophenone ${ }^{3}\left(n, \pi^{*}\right)$ & $\begin{array}{l}\text { isopropanol } \\
\text { cyclohexane } \\
\text { benzhydrol } \\
\text { isoborneol } \\
\text { p-F-phenol } \\
p-\text { - -phenol }\end{array}$ & $\begin{array}{r}400 \\
300 \\
300 \\
-250 \\
-3900 \\
-3900\end{array}$ & $\begin{array}{l}1.0 \mathrm{~b}, \mathrm{c} \\
1.0 \mathrm{~b}, \mathrm{c} \\
1.0 \mathrm{c} \\
0.9 \mathrm{c} \\
0.84 \mathrm{~b} \\
0.61\end{array}$ \\
\hline Acetophenone ${ }^{3}\left(n, \pi^{*}\right)$ & $\begin{array}{l}\text { isopropanol } \\
\text { I-phenylethanol }\end{array}$ & $\begin{array}{l}-800 \\
-900\end{array}$ & $\begin{array}{l}0.75 \mathrm{c} \\
0.60 \mathrm{c}\end{array}$ \\
\hline Biacety| $3\left(n, \pi^{*}\right)$ & isopropanol & 6200 & $1.0 \mathrm{c}$ \\
\hline Pheny Ibenzophenone $3\left(\pi, \pi^{*}\right)$ & isopropanol & 4000 & $1.0 \mathrm{C}$ \\
\hline
\end{tabular}

Data collected in ref 18 ; $b$ Radical intermediate; $c$ Final product. 
estimated from the change in $\mathrm{C}=0$ bond lengths betwen $S_{0}$ and $T_{1}$ and the ratio of $\nu \mathrm{CO}$ frequencies: the values $\left(S_{0}\right)-d\left(T_{1}\right)=0.12 A^{0}$ and $f_{S_{0}}=60 \times 10^{4} \mathrm{~cm}^{-1} A^{0-2}(18)$ were employed in all calculations.

Although the changes in $\phi$ are not very large, they can be made considerably more significant by use of heavy atoms to increase the probability of $\operatorname{lm}_{m} S_{0}$ crossing. Whereas the yields decrease when $E_{\omega}<E_{T_{1}}$, the yields remain invariant $(\phi=1)$ when $E_{\omega}>E_{T_{1}}(18)$.

Intramolecular photoabstractions of $\gamma$-hydrogen atoms in ketones can also be studied within the same formalism. The reactions are assumed to proceed via a cyclohexane-chair configuration and the effective force constants are $f_{r}=11.5 \times 10^{4} \mathrm{~cm}^{-1} A^{O-2}$ and $f_{p}=9.8 \times 10^{4} \mathrm{~cm}^{-1} A^{0-2}$ and $v=10^{13} \mathrm{~s}^{-1}(18)$. Table 2 presents the rates for tunnelling which reproduce the experimental values and the calculated rates for thermal activation with a preexponential factor of $6 \times 10^{12} \mathrm{~s}^{-1}$. For all the cases tunnelling seems more important than thermal activation, although the two mechanisms are of a comparable magniturle. Owing to the cyclohexane-chair configuration of the transition state the $\mathrm{O}$ and $\mathrm{H}$ atoms cannot approach so closely as in the linear configuration of the intermolecular process. Such distance is now ca. 2 AO to be compared with the bond length of $1.1 \mathrm{~A}$. According to the Pauling relationship, $n=n_{0}$ exp $\left(I-I_{0}\right) / a$ where $a=0.265 A^{\circ}$ (22). This increase in I leads virtually to zero bond order for the $O H$ bond at the transition state. Consequently the effective bond order for $\mathrm{CH}$ and $\mathrm{OH}$ bonds is now $n^{\neq}=0.25$ and through eqs (1) and (2) we can estimate $d X H \simeq 0.89 A^{\circ}$. This value together with the value previously found for the $\mathrm{CO}$ bond, $\mathrm{d}_{\mathrm{CO}}=0.308 \mathrm{~A}^{\circ}$ leads to an average $\mathrm{d} \simeq 0.6 \mathrm{~A}^{\mathrm{O}}$ in close agreement with the empirical values for this sort of reactions.

TABLE 2. Intramolecular $\gamma$-hydrogen photoabstractions in $\mathrm{CH}_{3} \mathrm{CO}\left(\mathrm{CH}_{2}\right)_{2} \mathrm{CHR}_{1} \mathrm{R}_{2}$ a

\begin{tabular}{|c|c|c|c|c|c|c|c|c|}
\hline $\mathbf{R}_{1}$ & $\mathbf{R}_{2}$ & State $\underline{d}$ & $d / A^{\circ}$ & tunnel l ing & $s^{-1}$ thermal & $\begin{array}{l}E_{1}-E_{e} / \mathrm{cm}^{-1} \text { e } \\
\text { polar medium }\end{array}$ & polar & $\underset{\text { nonpolar }}{\phi_{r}}$ \\
\hline H & $H \underline{b}$ & $3\left(n, \pi^{*}\right)$ & 0.60 & $1.3 \times 10^{7}$ & $1.0 \times 10^{7}$ & 500 & 0.85 & 0.43 \\
\hline H & $H \underline{b}$ & $1\left(n, \pi^{*}\right)$ & 0.61 & $1.8 \times 10^{8}$ & $6 \times 10^{6}$ & -2300 & 0.06 & 0.06 \\
\hline $\mathrm{CH}_{3}$ & $\mathrm{CH}_{3} \mathrm{C}$ & $3\left(n, \pi^{*}\right)$ & 0.605 & $3.2 \times 10^{8}$ & $1.5 \times 10^{8}$ & 100 & 1.00 & 0.49 \\
\hline $\mathrm{CH}_{3}$ & $\mathrm{CH}_{3} \mathrm{C}$ & $1\left(n, \pi^{*}\right)$ & 0.63 & $2.0 \times 10^{9}$ & $6 \times 10^{8}$ & -3300 & 0.27 & 0.24 \\
\hline
\end{tabular}

a Data of ref $18 ; \underline{b} D(C H)=409.5 \mathrm{~kJ} \mathrm{~mol}^{-1} ; \underline{c} \mathrm{D}(\mathrm{CH})=380.5 \mathrm{~kJ} \mathrm{~mol}^{-1} ; \underline{d} E\left(\mathrm{~S}_{1}\right)=28000 \mathrm{~cm}^{-1}, E\left(\mathrm{~T}_{1}\right)=26000$ $\mathrm{cm}^{1}$; $\mathrm{e}_{\mathrm{S}_{\mathrm{O}}}=48 \times 10^{4} \mathrm{~cm}^{-1} \mathrm{AO}^{-2}$.

Since tunnelling is estimated to be comparable to thermal activation for these reactions, it is important to assess experimentally this mechanism. A suggestion was made that the increase in the reaction yields with solvent polarity, can be attributed to an increase in the energy of the crossing point $\omega$, due to some small change in configuration of the ground state. Data of Table 2 seems to support such an idea. The fact that $\phi_{r}$ in a polar solvent is not unity for $\mathrm{CH}_{3} \mathrm{CO}\left(\mathrm{CH}_{2}\right)_{2} \mathrm{CH}_{3}$ can be attributed to some competition between tunnelling and thermal activation; from the experimental yields tunnelling seems to contribute to ca. $70 \%$ to the overall reaction. However the most clear proof of tunnelling comes from the study (18) of quantum yields of photoabstraction of valerophenone $n$, $\pi^{*}$ triplet in polar and nonpolar media (Table 3). In benzene where we expect $E_{\omega}<E_{T_{1}}$, because $\phi_{r}<1$, the saturation of the solution with xenon decreases the yield of reaction due to a more efficient i.s.c. at $\omega$. In ethanol, although the concentration of $X e$ in solution is virtually the same, no effect on $\phi_{r}=1.0$ was found, supporting the view that $E_{\omega}>E_{T_{1}}$ and that tunnelling is the dominant mechanism in this reaction.

TABLE 3. Quantum yields for the photoreduction of ${ }^{3}\left(n, \pi^{*}\right)$ valerophenone a

\begin{tabular}{lcc}
\hline Solvent & no gas & $\begin{array}{c}\text { saturated with } \\
\text { xenon }\end{array}$ \\
\hline Ethanol & 1.00 & 0.98 \\
Benzene & 0.44 & 0.18 \\
\hline
\end{tabular}

ą Data from ref. 18

All these data support the conclusions obtained from the theoretical calculations that H-photoabstraction reactions can be viewed as radiationless processes. Furthermore the potential energy barrier given by the classical intersecting-state-model seems to be adequate to deal with reactions of ground and excited states, either via thermal activation or via nuclear tunnelling. 


\section{$\alpha$-PHOTOCLEAVAGE OF KETONES}

The theoretical analysis of the photochemical $\alpha$-cleavage of ketones as radiationless transitions has been discussed in detail elsewhere(23). The reactivity towards $\alpha$-cleavage depends essentially on the strength of the $\mathrm{CO}-\mathrm{C}_{\alpha}$ bond and on the separation of the potential energy curves, d. For the construction of the potential energy curves the following vibrations were used: $\mathrm{C}=\mathrm{O}$ stretching, out - and in- plane wag and $\mathrm{C}=\mathrm{C}$ stretching in reactants and $\mathrm{C}=0$ stretch and bending in the radical products. Table 4 presents the results of the calculation for a few ketones in vapour phase where $d=0.63 A^{\circ}$ was treated as an empirical parameter.

TABLE 4. Calculated and experimental rates for $\alpha$-cleavage of $3\left(n, \pi^{*}\right)$ ketones aㅗ

\begin{tabular}{llrr} 
& Experimental & tunnelling & $\begin{array}{c}\mathrm{k} / \mathrm{s}^{-1} \\
\text { therma I activation }\end{array}$ \\
\hline Acetone & $6 \times 10^{2}$ & $4 \times 10^{2}$ & $2.5 \times 10^{2}$ \\
Butanone & $6 \times 10^{4}$ & $8 \times 10^{4}$ & $4 \times 10^{4}$ \\
3-Methylbutanone & $6 \times 10^{6}$ & $8 \times 10^{6}$ & $3 \times 10^{6}$ \\
\hline
\end{tabular}

a Data from ref. 23 with $A=6 \times 10^{12} \mathrm{~s}^{-1} ; f_{r}=19.5 \times 10^{4} \mathrm{~cm}^{-1} A^{-1}$ $f_{p}=4.7 \times 10^{4} \mathrm{~cm}^{-1} A^{0-2}$.

Here we will simply show that the $d$ value is a reasonable one, because the energy barrier is not entirely due to stretching vibrations, but has a small contribution of the bending modes. Furthermore, the best set of force constants employed in the study of more than 20 reactions implies also a small difference in the contributions of the $\mathrm{CC}$ and $\mathrm{CO}$ vibrations. For purely stretching motions with equal contributions of $C C$ and $C O$ bonds, the relevant lengths are $I_{C C}=1.54 A^{\circ}$ and $I_{C O}=(1.43+1.21) / 2$, average of a single and double $\mathrm{CO}$ bond lengths. Assuming again the conventional view of the $3\left(n, \pi^{*}\right)$ state as alkoxy radical like, the change in reaction bond orders is due to the breaking of a $\mathrm{CC}$ bond and the formation of a $\mathrm{CO}$ double bond from the single alkoxy $\mathrm{CO}$ bond. Consequently $\mathrm{n}^{\neq}=1 / 2$ and eqs (1) and (2) give $d=0.62 A^{\circ}$ very close to the empirical value, neglecting again any variation of $n$ with $\Delta \mathrm{E}$.

In the examples of Table 4 tunnelling is calculated to contribute by ca. $60-70 \%$ for the overall mechanism. In several other cases which probably involve some charge-transfer character and consequently smaller $d$ values but higher force constants, the contribution of tunnelling was found to be more significant with the exception of pentanone were the two rates are equal (23). For example with cyclopentanone the rate of tunnelling is more than two orders of magnitude higher than the one of thermal activation. The study of the efficiencies of these reactions is not as useful as with $\mathrm{H}$-abstractions to assess the tunnelling mechanism. The ketone ground state and the radical products have a surface touching. Whereas temperature does not alter significantly the energy content of the excited molecules that go over the energy barrier, it can alter significantly such an energy content for a tunnelling process from the thermal equilibrium population of the excited state. The probability of jump from products to $\mathrm{S}_{\mathrm{O}}$, in the touching region, will be higher when the velocity of crossing is smaller, i.e., when the energy content of the molecules is close to the energy of the surface touching. The yield of cleavage of acetone vapour is 1.0 at $154^{\circ} \mathrm{C}$ but decreases to 0.28 at $44^{\circ} \mathrm{C}(24)$; in these conditions there is no change in the triplet yield. This supports again the view that tunnelling is the dominant mechanism and other data in gas phase and solution support also this view for a few more compounds (23).

\section{PHOTOCYCLOADDITIONS OF KETONES TO OLEFINS}

Allowed photocycloadditions of excited ketones to olefins can also be studied within the formalism that we have been describing and again nuclear tunnelling has been found to be the dominant mechanism (25). As a typical illustrative case, the addition of $3\left(n, \pi^{*}\right)$ acetone to cis 3 -hexene involves the $C=0$ stretching and bending modes and the $\mathrm{CC}$ stretch in reactants and two $\mathrm{C}-\mathrm{O}$ and $\mathrm{C}-\mathrm{C}$ stretches in the products. The relevant point is that the experimental rate can be matched by a tunnelling mechanism, $\mathrm{k}=4 \times 10^{7} \mathrm{M}^{-1}$ $\mathrm{s}^{-1}$ with $d=0.737 \mathrm{~A}^{\circ}$.

$A$ rough estimation of $d$ can be made from the average ${ }^{I} C=C\left(1.375 A^{0}\right)$ and $\left.I_{C}-O^{(1.426} A^{0}\right)$ in reactants and $I_{C}-C\left(1.537 A^{\circ}\right)$ and $2 I_{C}-O$ in products. Under the assumption that one of the bonds, i.e. $C-O$, does not contribute to the overall bond order due to the large bending contribution, then $n^{\neq}=0.417$ and $d \simeq$ $0.72 \mathrm{AO}$ in reasonable agreement with the empirical value.

The calculated rate for thermal activation is only $3 \times 10^{3} \mathrm{M}^{-1} \mathrm{~s}^{-1}$ with $A=6 \times 10^{12} \mathrm{~s}^{-1}$. For these reactions the crossing between the biradical product and the ground state potential energy curves is found to be always below $T_{1}$, which does not allow an assessment of tunnelling through the study of reaction efficiencies. 


\section{CONCLUDING REMARKS}

The distinction between pure thermal activation and nuclear tunnelling processes, which have the same energy barrier, is not very clear when the temperature increases, because tunnelling from populated high vibrational levels becomes significant. However, the temperature where this effect becomes noticeable can be close or higher than room temperature when more than one vibrational mode is effective (26). This was the reason why the formalism of two distinct mechanisms was employed in this paper.

Common links between ground and excited state chemistry have been sought in the past with different amounts of sucess (27). The present classical intersecting-state-model seems to be another promising link, both from a quantitative and qualitative point of view. The establishment of potential energy barriers for ground and excited reactions within a common formalism, allows one to assess more easily the possible relevance of thermal activation or nuclear tunnelling as mechanisms of chemical reactions. Here we have shown through some selected examples that tunnelling can be an important mechanism for several photochemical reactions at room temperature. Such a mechanism is not restricted to light atoms, such as hydrogen and deuterium, but seems to be present in other situations where bonds between heavier atoms, such as carbon and oxygen are involved. We have not considered explicitly the role of entropy effects on photochemical reactivity (28). However, they were discussed, through a different formalism, for some conformational effects present on intramolecular $\mathrm{H}$-photoabstraction reactions.

\section{REFERENCES}

1 - A. R. Miller, J. Am. Chem. Soc., 100, 1984 (1978).

2 - J. R. Murdoch and D. E. Magnoli, J. Am. Chem. Soc., 103, 7465 (1981);

J. Donnella and J. R. Murdoch, J. Am. Chem. Soc., 106, 4724 (1984).

3 - E. Grunwald, J. Am. Chem. Soc., 107, 125 (1985).

4 - R. A. Marcus, J. Chem. Phys., 24, 966 (1956); J. Phys. Chem., 72, 891 (1968).

5 - A. J. C. Varandas and S. J. Formosinho, J. Chem. Soc., Chem. Commun., 163 (1986); J. Chem. Soc., Faraday Trans. 2, in publication.

6 - N. Agmon and R. D. Levine, Chem. Phys. Letters, 52, 197 (1977).

7 - S. J. Formosinho, Rev. Port. Quim., 27, 427 (1985).

8 - H. S. Johnston and C. Parr, J. Am. Chem. Soc., 85, 2544 (1963).

9 - R. B. Woodward and R. Hoffmann, "The Conservation of Orbital Symmetry", Academic Press, 1970.

10 - B. C. Garret, D. G. Truhlar, J. Am. Chem. Soc., 102, 2559 (1980); P. Pechukas, Ber. Bunsenges.

Phys. Chem., 86, 372 (1982), E. Pollak and R. D. Levine, ibid, 86, 458 (1982), D. G. Truhlar, B. C. Garret, Ann. Rev. Phys. Chem., 35, 159 (1984).

11 - J. R. Murdoch, J. Am. Chem. Soc., 105, 2660 (1983).

12 - N. Agmon, J. Am. Chem. Soc., 106, 6960 (1984).

13 - S. J. Formosinho, to be published.

14 - J. Ulstrup and J. Jortner, J. Am. Chem. Soc., 101, 3744 (1979); Chem. Phys. Letters, 63,236 (1979).

15 - S. J. Formosinho, J. Chem. Soc., Faraday Trans. 2, 72,1313 (1976); 73, 1978 (1978).

16 - G. D. Abhot and D. Phillips, Mol. Photochem., 8, 289 (1977).

17 - J. Sühnel and K. Gustav, Mol. Photochem., 8, 437 (1977); G. St. Nikolov and P. Markov, J. Photochem., 16, 93 (1981)

18 - L. G. Arnaut, S. J. Formosinho and A. M. da Silva, J. Photochem., 27, 135 (1984).

19 - V. Schomaker and D. P. Stevenson, J. Am. Chem. Soc., 63, 37 (1941).

20 - K. H. Grellmann, H. Weller and E. Tauer, Chem. Phys. Letters, 95, 195 (1983).

21 - L. Salem, J. Am. Chem. Soc., 96, 3486 (1974), N. J. Turro and V. Ramamurthy, Mol. Photochem., 8, 239 (1977).

22 - L. Pauling, J. Am. Chem. Soc., 69, 542 (1947).

23 - L. G. Arnaut and S. J. Formosinho, J. Photochem., 31, 315 (1985).

24 - H. E. O'Neal and C. W. Larson, J. Phys. Chem., 73, 1011 (1969).

25 - L. G. Arnaut and S. J. Formosinho, to be published.

26 - J. Jortner, J. Chem. Phys., 64, 4860 (1976); E. Buhks and J. Jortner, J. Phys. Chem., 84, 3370 (1980), S. J. Formosinho and M. G. M. Miguel, J. Chem. Soc., Faraday Trans. 1, 80, 1745 (1984).

27 - J. Michl, Mol. Photochem., 4, 243, 257, 287 (1972); L. Salem, Science, 191, 822 (1976); N. D. Epiotis, Pure Appl. Chem., 51, 203 (1979); H. E. Zimmerman, Topics Current Chem., 100, 45 (1982).

28 - F. Gessner and J. C. Scaiano, J. Am. Chem. Soc., 107, 7206 (1985). 